\author{
Alessandra Cherchi \\ Erminia Stochino \\ Maria Paola Piccardi \\ Maria Del Zompo
}

\section{Role of dopaminergic system in migraine}

A. Cherchi • E. Stochino • M.P. Piccardi

M. Del Zompo (两)

Headache Center

B.B. Brodie Department of Neurosciences

University of Cagliari

P.O. San Giovanni di Dio

Via Ospedale 46, I-09124 Cagliari, Italy

e-mail: delzompo@unica.it

Tel.: +39-070-6092438

Fax: +39-070-653584

\begin{abstract}
The theory that hypersensitivity of dopamine (DA) system is involved in the pathogenesis of migraine has been supported by various authors on the basis of clinical, pharmacological and, recently, genetic evidence. Apomorphine, a selective and specific DA agonist, has a cerebral vasodilatatory effect and increases blood flow significantly in the middle cerebral artery in migraineurs. Processes from central DA neurons terminate in close contact with penetrating arterioles and cerebral capillaries in the cerebral cortex. This finding reaffirms the role of central neurogenic mechanisms in the regulation of the cerebral circulation and, we believe, fur-
\end{abstract}

ther supports the major role of dopamine in the neurogenic mechanisms of migraine. Various studies have been carried out to verify the involvement of DA in migraine pathogenesis using molecular genetics as a tool. A positive association between the "dopaminergic" phenotype of migraine without aura and the $\mathrm{D} 2$ receptor gene has been found. To explain dopaminergic hypersensitivity in migraine without aura, we will study the genes encoding proteins involved in the signal transduction system.

Key words Dopamine $\cdot$ Migraine pathogenesis $\cdot$ Dopaminergic hypersensitivity $\cdot$ Genetics

\section{Introduction}

Despite recent advances, the pathogenesis of migraine is still unclear. The study of migraine pathophysiology includes the investigation of neurotransmitters and their potential role in the development of the attack. Scientific and clinical interest has focused on the role of 5-hydroxytryptamine (5-HT). However, the dopaminergic system also appears to play a major, still less recognized, role in migraine and dopamine (DA) can be considered the "second putative protagonist in headache" [1]. Several arguments suggest the involvement of DA in migraine pathophysiology $[2,3]$.

The dopaminergic system has a prominent role in motor and sensorial control, in modulating cognitive processes, and in the control of both prolactin and growth hormone secretion. Recently, a key role in the regulation of cerebral microcirculation has been demonstrated [4]. Based on their structure and pharmacological characteristics, five subtypes of DA receptors have been described: D1, D4 and D5 are Gs protein-coupled receptors, D2 is a Gi protein-coupled receptor, and the signal transducing system of D3 is not yet completely characterized.

\section{Evidence for hypersensitivity of the dopaminergic system in migraine}

The theory that hypersensitivity of the DA system is involved in the pathogenesis of migraine has been supported by various authors on the basis of clinical, pharmacological and, recently, genetic evidence. 
Clinical evidence of DA involvement was described by Blau, who observed intense yawning, craving for some foods and altered sensorial perception during the initial phase of the migraine attack [5]. Yawning is a behavior controlled by various neurotransmitters and neuropeptides, which act at the level of the periventricular hypothalamic nucleus. Among the neurotransmitters which elicit yawning, DA plays a major role, acting through postsynaptic D2 receptors located in cell bodies of oxytocinergic neurons present in the periventricular hypothalamic nucleus and causing the release of oxytocin and subsequent yawning [6].

In 1991, Blin et al. [7] performed a controlled study to evaluate the reaction of migraineurs to apomorphine, a selective and specific DA agonist that causes yawning in healthy volunteers. The drug was administered at low dosage to a group of 9 migraineurs and 9 healthy volunteers. The migraineurs exhibited a statistically significant enhanced response to the apomorphine challenge, supporting the existence of DA hypersensitivity [7].

Nausea and vomiting are partly controlled by the activation of DA system through D2 receptors located in the "chemoreceptor trigger zone" and in the nucleus of solitary tract in the brainstem. Dysosmia indicates an activation of the olfactory tubercle, which is particularly rich in dopaminergic fibers. The administration of apomorphine to a migraineur caused an episode of nausea, sweating, yawning, hypotension, vomiting and syncope at a dosage that is ineffective in non-migraineurs, further suggesting the existence of DA hypersensitivity [2]. Hypotension and occasionally syncope have been associated with migraine [10]. This behavior is probably mediated by a hyperresponsiveness of DA receptors located on presynaptic noradrenergic sympathetic ganglia, and their stimulation inhibits the norepinephrine release from sympathetic nerve terminals.

\section{Dopamine and cerebral blood flow}

Apomorphine has a cerebral vasodilatatory effect and increases blood flow significantly in the middle cerebral artery in migraineurs [8]. This increase has been confirmed in a controlled study versus placebo [9], supporting the hypothesis of DA hypersensitivity in migraine. DA receptors have been located directly in cerebral arteries that are believed to be involved in the pathogenesis of migraine. In fact, DA receptors have been localized to pial vessels [11], the site of neurogenic inflammation believed to play a role in the pathogenesis of migraine. Cortical blood vessels are innervated by axons containing norepinephrine, acetylcholine, serotonin and several peptides.
Recently, it has been demonstrated that dopaminergic terminals are closely apposed to microvessels within the cortex and that exogenous dopamine, when iontophoresed into microvessels, causes them to constrict [4]. However, in the presence of serotoninergic and adrenergic receptor blockers, this amine produces vasodilatation, suggesting that DA acts as an agonist on adrenergic and serotoninergic receptors [12]. This finding reveals a new vasomotor role for dopamine, possibly a direct involvement of DA neurotransmission in the regulation of local cerebral cortical blood flow.

Using immunocytochemical techniques to visualize dopaminergic terminals, the authors demonstrated that processes from central DA neurons terminate in close contact with penetrating arterioles and cerebral capillaries in cerebral cortex. This discovery provides morphological evidence of a direct interaction between central perivascular terminals and cerebral microvessels. This finding reaffirms the role of central neurogenic mechanisms in the regulation of the cerebral circulation. Nerve fibers originating from cranial autonomic and sensory ganglia, mainly sphenopalatine, trigeminal and superior cervical ganglia, innervate intracranial cerebral arteries; through neurotransmitters and peptides, these nerve fibers can either constrict or dilate arteries and arterioles. Given the diffuse nature of the innervation and the relatively large size of the vessels innervated, extrinsic perivascular nerves are unlikely to play a role in the highly localized changes in flow initiated by functional brain activity [12]. We believe that this finding further supports the major role of dopamine in the neurogenic mechanisms involved in migraine.

\section{Dopamine antagonist in migraine therapy}

Dopaminergic antagonists have been proposed as antimigraine drugs [13-15]. A significant amount of evidence exists to support the efficacy of D2 antagonists in the acute treatment of migraine. In fact, haloperidol, prochlorperazine, domperidone and chloropromazine are all efficacious in the treatment of migraine attacks [3]. Moreover, flunarizine, a calcium channel blocker that displays significant DA antagonist properties, is a potent drug in preventive therapy of migraine.

\section{Genetic studies}

The prevalence of migraine among members of the same family suggests the presence of a genetic component for this disorder. We consider migraine to be a complex disease 
caused by a combination of various environmental and genetic factors, such as epilepsy or diabetes. Various studies have been carried out to verify the involvement of DA in migraine pathogenesis using molecular genetics as a tool. A study performed on a sample of 250 patients affected by migraine with aura considered the gene for the $\mathrm{D} 2$ receptor to be a candidate gene [16].

Another study was performed by our group [17], using a family-based method, in a randomized sample of 50 trios, each trio consisting of a subject affected by migraine without aura and his or her parents. In this study, we analyzed the genes for D1, D2, D3 and D4 receptors. We found a positive association between the "dopaminergic" phenotype of migraine without aura and the D2 receptor gene [17]. Recently, we studied D5 in 100 trios and concluded that it was not involved in migraine pathogenesis in the study population [18].

Dopaminergic hypersensitivity may be related not only to an altered receptor function, but also to an abnormal functioning of the signal transduction pathways. The dopaminergic receptors are coupled to GTP-binding proteins, can modulate phosphoinositide pathways and regulate the activity of potassium and calcium channels. To further explain the dopaminergic hypersensitivity in migraine without aura, we will study the genes encoding the proteins involved in the signal transduction system.

\section{References}

1. Sicuteri F (1977) Dopamine, the second putative protagonist in headache. Headache 17:129-131

2. Del Zompo M, Lai M, Loi V, Pisano MR (1994) Dopamine hypersensitivity in migraine: role in apomorphine syncope. Headache 35:222-224

3. Peroutka SJ (1997) Dopamine and migraine. Neurology 49:1-6

4. Krimer SK, Muly III EC, Williams GV, Goldman-Rakic PS (1998) Dopaminergic regulation of cerebral cortical microcirculation. Nat Neurosci $1: 263-265$

5. Blau JN (1992) Migraine: theories of pathogenesis. Lancet 339:1202-1206

6. Argiolas A, Melis MR (1998) The neuropharmacology of yawning. Eur $\mathbf{J}$ Pharmacol 343:1-16

7. Blin O, Azulay JP, Masson G, Aubrespy G, Serratrice G (1991) Apomorphineinduced yawning in migraine patients: enhanced responsiveness. Clin Neuropharmacol 14:91-95
8. Mc Culloch J, Harper AM (1977) Cerebral circulation: effect of stimulation and blockade of dopamine receptors. Am J Physiol 233:222-227

9. Piccini P, Pavese N, Palombo C, Pittella G et al (1995) Transcranial Doppler ultrasound in migraine and tension-type headache after apomorphine administration: double-blind crossover versus placebo study. Cephalalgia 15:399-403

10. Bickerstaff ER, Birm MD (1961) Impairment of consciousness in migraine. Lancet ii:1057-1059

11. Edvinsson L, McCulloch J, Sharkey J (1985) Vasomotor responses of cerebral arterioles in situ to putative dopamine receptor agonists. $\mathrm{Br} \mathrm{J}$ Pharmacol 85:403-410

12. Iadecola C (1998) Neurogenic control of the cerebral microcirculation: is dopamine minding the store? Nat Neurosci 1:263-265

13. Yealy DM (1994) Acute pain management. Acad Emerg Med 1:186-189
14. Kelly AM, Ardagh M, Curry C, D'Antonio J, Zebic S (1997) Intravenous chloropromazine versus intramuscular sumatriptan for acute migraine. J Accid Emerg Med 14:209-211

15. William EM et al (1997) Guidelines for the diagnosis and management of migraine in clinical practice. CMAJ 156:1273-1287

16. Peroutka SJ, Wilhoit T, Jones K, (1997) Clinical susceptibility to migraine with aura is modified by dopamine D2 receptor (DRD2) NcoI alleles. Neurology 49:201-206

17. Del Zompo M, Cherchi A, Palmas MA, Ponti M, Bocchetta A, Gessa GL, Piccardi MP (1998) Association between dopamine receptor genes and migraine without aura in a Sardinian sample. Neurology 51:781-786

18. Palmas MA, Cherchi A, Stochino E, Congiu D, Del Zompo M (2000) Dopamine genes and migraine. $\mathrm{J}$ Headache Pain 1:S153-S156 